\title{
Minimum Cost Homomorphisms with Constrained Costs
}

\author{
Pavol Hell ${ }^{1}$ and Mayssam Mohammadi Nevisi ${ }^{1}$ \\ School of Computing Science, Simon Fraser University, Burnaby, Canada, \\ pavol, mayssamm@sfu.ca*
}

\begin{abstract}
The minimum cost homomorphism problem is a natural optimization problem for homomorphisms to a fixed graph $H$. Given an input graph $G$, with a cost associated with mapping any vertex of $G$ to any vertex of $H$, one seeks to minimize the sum of costs of the assignments over all homomorphisms of $G$ to $H$. The complexity of this problem is well understood, as a function of the target graph $H$. For bipartite graphs $H$, the problem is polynomial time solvable if $H$ is a proper interval bigraph, and is NP-complete otherwise. In many applications, the costs may be assumed to be the same for all vertices of the input graph. We study the complexity of this restricted version of the minimum cost homomorphism problem. Of course, the polynomial cases are still polynomial under this restriction. We expect the same will be true for the NP-complete cases, i.e., that the complexity classification will remain the same under the restriction. We verify this for the class of trees. For general graphs $H$, we prove a partial result: the problem is polynomial if $H$ is a proper interval bigraph and is NP-complete when $H$ is not chordal bipartite.
\end{abstract}

Keywords: homomorphisms, NP-completeness, dichotomy

\section{Introduction}

Suppose $G$ and $H$ are graphs (without loops or multiple edges). A homomorphism $f: G \rightarrow H$ is a mapping $V(G) \rightarrow V(H)$ such that $f(u) f(v) \in E(H)$ whenever $u v \in E(G)$. For a fixed graph $H$, a number of computational problems have been considered. In the homomorphism problem, one asks whether or not an input graph $G$ admits a homomorphism to $H$. It is known that this problem is polynomial time solvable if $H$ is bipartite, and is NP-complete otherwise [1. In the list homomorphism problem, the input graph $G$ is equipped with lists (sets) $L(x) \subseteq V(H)$, for all $x \in V(G)$, and one asks whether or not there exists a homomorphism $f: G \rightarrow H$ with $f(x) \in L(x)$ for all $x \in V(G)$. This problem is known to be polynomial time solvable if $H$ is an interval bigraph,

\footnotetext{
* This work was partially supported by NSERC (Canada) and by ERCCZ LL 1201 Cores (Czech Republic); it was done in part while the first author was visiting the Simons Institute for the Theory of Computing
} 
and is NP-complete otherwise [2]. (An interval bigraph is a bipartite graph $H$ with parts $X$ and $Y$ such that there exist intervals $I_{x}, x \in X$, and $J_{y}, y \in Y$, for which $x y \in E(H)$ if and only if $I_{x} \cap J_{y} \neq \emptyset$.) In this paper we address the minimum cost homomorphism problem, in which the input graph is equipped with a cost function $c: V(G) \times V(H) \rightarrow \mathbb{N}$ and one tries to minimize the total cost $\sum_{u \in V(G)} c(u, f(u))$. Minimum cost homomorphism problems were introduced in [3]. They were motivated by an application in repair and maintenance scheduling; however, the problem arises in numerous other contexts, e.g. in the minimum colour sum problem and the optimum cost chromatic partition problem 4/5. To state it as a decision problem, the input includes an integer $k$, and one asks whether or not there exists a homomorphism of total cost at most $k$. This problem is known to be polynomial time solvable if $H$ is a proper interval bigraph, and is NP-complete otherwise [6]. (An interval bigraph is a proper interval bigraph if the above two families of intervals $I_{x}, x \in X$, and $J_{y}, y \in Y$ can be chosen to be inclusion-free, i.e., no $I_{x}$ properly contains another $I_{x^{\prime}}$ and similarly for the $J_{y}$ 's.)

These results are dichotomies in the sense that for each $H$ the problem is polynomial time solvable or NP-complete. They have subsequently been studied in more general contexts, for graphs with possible loops, for digraphs, and for general relational structures (in the context of constraint satisfaction problems). In particular, there is a dichotomy for the homomorphism problem for graphs with possible loops [1, but dichotomy is only conjectured for digraphs (and more general structures) 7/8. A dichotomy for list homomorphism problems for graphs with possible loops was established in [2], then a general dichotomy was proved for all relational systems in [10. (A more structural dichotomy classification for digraphs was given in [12.) For minimum cost homomorphism problems, a dichotomy for graphs with possible loops is given in [․ . A structural dichotomy classification for digraphs was conjectured in [3, and proved in [11 (cf. 1314]). Then a general dichotomy for all relational systems was proved in [15]. Even more general dichotomy results are known, for so-called finite valued constraint satisfaction problems [16.

It is easy to see that minimum cost homomorphism problems generalize list homomorphism problems, which in turn generalize homomorphism problems. Minimum cost homomorphism problems also generalize two graph optimization problems, the minimum colour sum problem, and the optimum cost chromatic partition problem [45]. In the former, the cost function has only two values, 0 and 1 (and $k=0$ ). In the latter, the cost function is assumed to be constant across $V(G)$, i.e., $c(x, u)=c(u)$ for all $x \in V(G)$. This restriction, that costs only depend on vertices of $H$, appears quite natural even for the general minimum cost homomorphism problems, and appears not have been studied. In this paper we take the first steps in investigating its complexity.

Let $H$ be a fixed graph. The minimum constrained cost homomorphism problem for $H$ has as input a graph $G$, together with a cost function $c: V(H) \rightarrow \mathbb{N}$, and an integer $k$, and asks whether there is a homomorphism $f: G \rightarrow H$ of total $\operatorname{cost} \operatorname{cost}(f)=\sum_{u \in V(G)} c(f(u)) \leq k$. 
It appears that the added constraint on the cost function may leave the dichotomy classification from [6] unchanged; in fact, we can show it does not change it for trees $H$ (and in some additional cases, cf. Lemma 8 below.)

Theorem 1. Let $H$ be a fixed tree. Then the minimum constrained cost homomorphism problem to $H$ is polynomial time solvable if $H$ is a proper interval bigraph, and is NP-complete otherwise.

We believe the same may be true for general graphs $H$. We have obtained the following partial classification.

Theorem 2. Let $H$ be a fixed graph. Then the minimum constrained cost homomorphism problem to $H$ is polynomial time solvable if $H$ is a proper interval bigraph, and is NP-complete if $H$ is not a chordal bipartite graph.

Of course, the first statement of the theorem follows from [6]. Only the second claim, the NP-completeness, needs to be proved. A bipartite graph $H$ is chordal bipartite if it does not contain an induced cycle of length greater than four. Both chordal bipartite graphs and proper interval bigraphs can be recognized in polynomial time [17/18. Proper interval bigraphs are a subclass of chordal bipartite graphs, and Lemma 8 below gives a forbidden subgraph characterization of proper interval bigraphs within the class of chordal bipartite graphs.

Our NP-completeness reductions in the proofs of Theorems 1 and 2 use a shorthand, where vertices $v$ of the input graph $G$ have weights $w(v)$. Adding polynomially bounded vertex weights does not affect the time complexity of our problems. Let $G, H$ be graphs, and, for every $v \in V(G)$ and every $i \in$ $V(H)$, let $c_{i}(v)$ denote the cost of mapping $v$ to $i$. Let $w: V(G) \rightarrow \mathbb{N}$ be a weight function. The weighted cost of a homomorphism $f: G \rightarrow H$ is $\operatorname{cost}(f)=$ $\sum_{v \in V(G)} w(v) \cdot c_{f(v)}(v)$. In the weighted minimum cost homomorphism problem for a fixed graph $H$, the input is a graph $G$, together with cost functions $c_{i}$ : $V(G) \rightarrow \mathbb{N}$ (for all $i \in V(H)$ ), vertex weights $w: V(G) \rightarrow \mathbb{N}$, and an integer $k$; and the question is if there is a homomorphism of $G$ to $H$ of weighted cost at most $k$.

The variant with constrained costs is defined similarly: the weighted minimum constrained cost homomorphism problem for $H$ has as input a graph $G$, cost function $c: V(H) \rightarrow \mathbb{N}$, vertex weights $w: V(G) \rightarrow \mathbb{N}$, and an integer $k$, and it asks if there is a homomorphism $f: G \rightarrow H$ with cost $\sum_{v \in V(G)} w(v) \cdot c(f(v)) \leq k$.

Clearly, when $w$ is a polynomial function, the weighted minimum cost homomorphism problem and the minimum cost homomorphism problem are polynomially equivalent. It turns out that this is also the case for the problems with constrained costs.

Theorem 3. Let $H$ be a fixed graph. The minimum constrained cost homomorphism problem to $H$ and the weighted minimum constrained cost homomorphism problem to $H$ with polynomial weights are polynomially equivalent. 


\section{Chordal Bipartite Graphs}

In this section, we investigate the minimum constrained cost homomorphism problem for graphs $H$ that contain an even cycle of length at least six as an induced subgraph. First we treat the case of hexagon, then we handle longer cycles.

Lemma 4. Let $H$ be a graph which contains hexagon as an induced subgraph. Then, the weighted minimum constrained cost homomorphism problem to $H$ is NP-complete.

For a fixed graph $H$, the pre-colouring extension problem to $H$ takes as input a graph $G$ in which some vertices $v$ have been pre-assigned to images $f(v) \in$ $V(H)$ (we say $v$ is pre-coloured by $f(v)$ ), and asks whether or not there exists a homomorphism $f: G \rightarrow H$ that extends this pre-assignment. This can be viewed a special case of the list homomorphism problem to $H$ (all lists are either singletons or the entire set $V(H)$ ), and has been studied under the name of OneOr-All list homomorphism problem, denoted $\operatorname{OAL}-\operatorname{HOM}(H)$ [2]. Here we adopt the abbreviation $\mathrm{OAL}-\mathrm{HOM}(H)$ for the pre-colouring extension problem.

The problem OAL-HOM $(H)$ was first studied in [192].

Lemma 5. [2] Let $C$ be a cycle of length $2 k$ with $k \geq 3$. Then the pre-colouring extension problem to $C$ is $N P$-complete.

We can now present the proof of Lemma 4

Proof. The membership in NP is clear. Let $C=1,2, \cdots, 6$ denote the hexagon and $h_{1} h_{2} \cdots h_{6}$ be an induced subgraph of $H$ which is isomorphic to $C$. We reduce from the pre-colouring extension homomorphism problem to $C$.

Let $(G, L)$ be an instance of OAL-HOM $(C)$, i.e., $G$ is a bipartite graph with $n \geq 2$ vertices and $m \geq 1$ edges, and some vertices $v$ of $G$ have been preassigned to $f(v) \in V(C)$. We construct an instance $\left(G^{\prime}, c, w, T\right)$ of the weighted minimum constrained cost homomorphism problem to $H$ as follows. The graph $G^{\prime}$ is a bipartite graph obtained from a copy of $G$, by adding, for every vertex $v \in V(G)$ pre-coloured $k$, a gadget that is the cartesian product of $v$ and the hexagon, using six new vertices $(v, 1),(v, 2), \cdots,(v, 6)$, and six new edges $(v, 1)(v, 2),(v, 2)(v, 3), \cdots,(v, 6)(v, 1)$. We also connect $v$ to exactly two neighbours of $(v, k)$ in its corresponding gadget. A vertex $v$ and its corresponding gadget is illustrated in Figure 1 .

We define the vertex weight function $w$ as follows.

- for every vertex $v$ in the copy of $G$, let $w(v)=1$

- for every pre-coloured vertex $v \in V(G)$ :

- $w((v, 1))=w((v, 4))=5 \times 36 n^{3}+1$,

- $w((v, 2))=w((v, 5))=1$,

- $w((v, 3))=36 n^{2}$,

- $w((v, 6))=6 n$. 


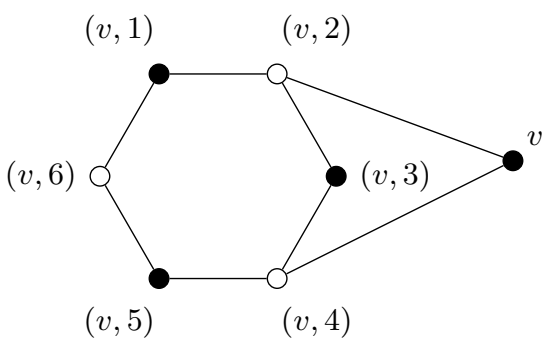

Fig. 1. A gadget in $G^{\prime}$ for a vertex $v \in V(G)$ pre-coloured by 3

We define the homomorphism cost function $c$ as follows.

$-c\left(h_{1}\right)=c\left(h_{4}\right)=0$,

$-c\left(h_{2}\right)=c\left(h_{5}\right)=36 n^{2}$,

$-c\left(h_{3}\right)=1$,

$-c\left(h_{6}\right)=6 n$,

$-c\left(h_{i}\right)=5 \times 36 n^{3}+1$ for all other vertices $h_{i} \in V(H)$.

Finally, we set $T=5 \times 36 n^{3}=180 n^{3}$.

We now claim that there is an extension of the pre-colouring $f$ to a homomorphism of $G$ to $C$ if and only if there is a homomorphism of $G^{\prime}$ to $H$ with weighted cost at most $T$.

First, assume that the pre-colouring can be extended to a homomorphism $f: G \rightarrow C$. We define a homomorphism $g: G^{\prime} \rightarrow H$ as follows.

$-g(u)=h_{i}$ iff $f(u)=i$ for every vertex $u \in V(G)$ and every $1 \leq i \leq 6$,

$-g((u, i))=h_{i}$ for every vertex $u \in V(G)$ pre-coloured $k$ and every $1 \leq i \leq 6$.

Claim. The function $g$ is a homomorphism of $G^{\prime}$ to $H$. Moreover, it only maps vertices of $G^{\prime}$ to the copy of $C$ in $G$, i.e., $g$ only uses vertices $h_{1}, h_{2}, \cdots, h_{6}$.

To prove the above claim, we distinguish three types of edges in $G^{\prime}$.

1. Edges $u v$ corresponding to the edges in $G(u, v \in V(G))$ : These are clearly mapped to edges in $H$ by $g$ as $g(u)=f(u)$ for all vertices $u \in V(G)$ and $f$ is a homomorphism of $G$ to $C$.

2. Edges $(u, i)(u, i+1)$ that connect two vertices of the gadgets: These edges map to the corresponding edge $h_{i} h_{i+1}$ by definition of $g$ (indices modulo 6 ).

3. Edges that connect a vertex $u \in V(G)$ to two vertices in its corresponding gadget: Notice that there is a gadget for $u$ in $G^{\prime}$ only when $u$ is pre-coloured $i$. So, we have $f(u)=i$. This further implies that $g(u)=h_{i}$. Also, notice that $g((u, i-1))=h_{i-1}$ and $g((u, i+1))=h_{i+1}$ by the definition of $g$ (again, all indices modulo 6). Hence, edges $u(u, i-1)$ and $u(u, i+1)$ also map to edges $h_{i-1} h_{i}$ and $h_{i} h_{i+1}$, respectively. 
This completes the proof of the above Claim. We now show that the cost of $g$ is at most $T=180 n^{3}$.

- For every vertex $u \in V(G), w(u)=1$ and $c(g(u)) \leq 36 n^{2}$. Also, there are exactly $n$ such vertices in $G^{\prime}$. This contributes at most $36 n^{3}$ to the cost of the homomorphism.

- For every pre-coloured vertex $u \in V(G)$, its corresponding gadget contributes exactly $4 \times 36 n^{2}$ :

- vertices $(u, 1)$ and $(u, 4)$ do not contribute, as $c\left(h_{1}\right)=c\left(h_{4}\right)=0$,

- vertices $(u, 2)$ and $(u, 5)$ each contribute $36 n^{2}$,

- vertices $(u, 3)$ and $(u, 6)$ each contributes $36 n^{2}=6 n \times 6 n=36 n^{2} \times 1$.

There are at most $n$ gadgets in $G^{\prime}$ (one for every vertex $u \in V(G)$ ), and so, the total contribution of all vertices of the gadgets is at most $4 \times 36 n^{3}$. Therefore, the cost of $g$ is at most $5 \times 36 n^{3}=180 n^{3}=T$.

Conversely, let $g$ be a homomorphism of $G^{\prime}$ to $H$ which costs at most $T$. We prove that there is a homomorphism $f: G \rightarrow C$ extending the pre-colouring. First, we show that $g$ has the following two properties.

- It only maps vertices of $G^{\prime}$ to the vertices of the hexagon $h_{1}, h_{2}, \cdots, h_{6}$,

- all gadgets are mapped identically to the hexagon in $H$, that is, for all precoloured vertices $u \in V(G)$ and for every $1 \leq i \leq 6, g((u, i))=h_{i}$.

The first property holds because $c(a)>T$ for every vertex $a \in V(H)$ other than the vertices of the hexagon (and the fact that, by definition, all vertex weights are positive integers). In fact, we must have $w(u) \times c(g(u)) \leq T$, or equivalently, $c(g(u))<\frac{(T+1)}{w(u)}$, for every vertex $u \in V\left(G^{\prime}\right)$. This restricts possible images of vertices with large vertex weights. Consider vertices in the gadget of a vertex $u \in V\left(G^{\prime}\right)$. For instance, every $(u, 4)$ must map to either $h_{1}$ or $h_{4}$. Similarly, none of the $(u, 3)$ vertices can map to any vertex other than $h_{1}, h_{3}$, or $h_{4}$. Given that $(u, 3)$ and $(u, 4)$ are adjacent in $G^{\prime}$, their images must also be adjacent in $H$. This enforces $f((u, 3))=h_{3}$ and $f((u, 4))=h_{4}$ (for every $u$ that has a gadget in $\left.G^{\prime}\right)$. Similar to $(u, 4), g$ must also map every $(u, 1)$ to either $h_{1}$ or $h_{4}$, but $g((u, 1))=h_{4}$ is not feasible as it does not leave any options for the image of $(u, 2)$. Hence, $g((u, 1))=h_{1}$. This further implies that $g((u, 6))=h_{6}$ (as it is adjacent to $(u, 1)$ ), and finally, $g((u, 2))=h_{2}$ and $g((u, 5))=h_{5}$.

It is now easy to verify that for every vertex $u \in V(G)$ pre-coloured $j$, we always have $g(u)=h_{j}$. This is because $u$ is adjacent to $(u, j-1)$ and $(u, j+1)$ in $G^{\prime}$ and the only vertex in $H$ that is adjacent to the $g((u, j-1))=h_{j-1}$ and $g((u, j+1))=h_{j+1}$ and the cost of mapping to it is less than or equal to $T$ is $h_{j}$. This completes the proof as we can define a homomorphism $f: G \rightarrow C$ extending the pre-colouring by setting $f(v)=i \Longleftrightarrow g(v)=h_{i}$.

A shorthand of the construction used in the above proof is shown in Figure2. We now extend Lemma 4 to larger even cycles.

Lemma 6. Let $H$ be a bipartite graph which contains a cycle of length at least eight as an induced subgraph. Then the weighted minimum constrained cost homomorphism problem to $H$ is NP-complete. 

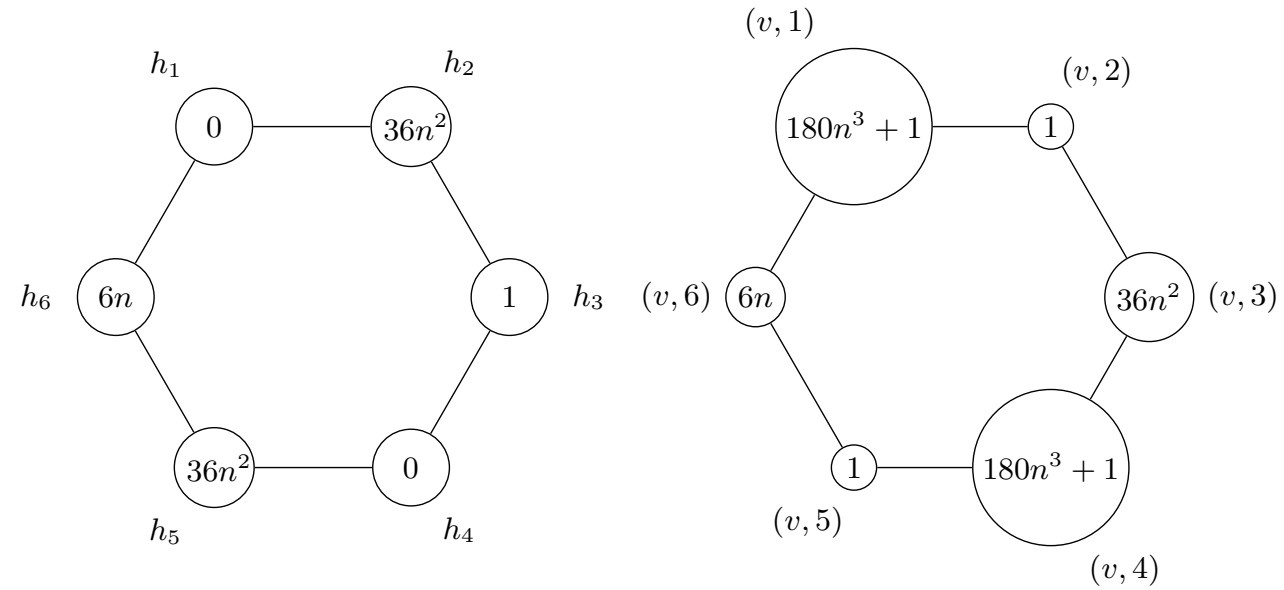

Fig. 2. A hexagon in $H$ together with associated homomorphism costs (left), and a gadget in $G^{\prime}$ together with vertex weights (right).

Proof sketch. The proof is similar to the proof of Lemma 4. We only discuss the reduction here. Let $C=1,2, \cdots, 2 k$ be an even cycle, and $h_{1} h_{2} \cdots h_{2 k}$ be an induced subgraph of $H$ which is isomorphic to $C(k \geq 4)$. Again, we reduce from OAL-HOM $(C)$. We take an instance of the OAL-HOM $(C)$, i.e., a graph $G$ with $n \geq 2$ vertices and $m \geq 1$ edges, with some vertices of $G$ pre-coloured by vertices of $C$. We construct a corresponding instance $\left(G^{\prime}, c, w, T\right)$ of the weighted minimum constrained cost homomorphism problem to $H$.

The graph $G^{\prime}$ is constructed exactly as before: we start with a copy of $G$ and for every vertex $v$ pre-coloured by $t$, we add the cartesian product of $v$ and $C$ using $2 k$ new vertices and $2 k$ new edges. Finally, make $v$ adjacent to two vertices in its corresponding gadget, $(v, t-1)$ and $(v, t+1)$ (all indices modulo $2 k)$.

We define the vertex weight function $w$ as follows.

- for every vertex $v$ in the copy of $G$, let $w(v)=1$

- for every pre-coloured vertex $v \in V(G)$ :

- $w((v, 1))=w((v, 4))=50 k n^{2}$,

- $w((v, 2))=w((v, 3))=w((v, 5))=1$,

- $w((v, i))=9 n$ for all $6 \leq i \leq 2 k$

We define the homomorphism cost function $c$ as follows.

- $c\left(h_{1}\right)=c\left(h_{4}\right)=0$,

- $c\left(h_{2}\right)=c\left(h_{3}\right)=c\left(h_{5}\right)=8 k n$,

- $c\left(h_{i}\right)=1$ for all $6 \leq i \leq 2 k$,

$-c\left(h_{i}\right)=50 k n^{2}$ otherwise. 
Finally, we set $T=50 k n^{2}-1$. As in the proof of Lemma 4, we argue that there is a homomorphism of $G$ to $C$ extending the pre-colouring if and only if there is a homomorphism of $G^{\prime}$ to $H$ with cost at most $T$.

This completes the proof of Theorem 2, as chordal bipartite graphs have no induced cycles of length greater than four.

We note that Theorem 2 gives only a partial dichotomy for the minimum constrained cost homomorphism problem, as there is a gap between the class of chordal bipartite graphs and the class of proper interval bigraphs. Specifically, the following result clarifies the gap.

Lemma 7. [20] A chordal bipartite graph $H$ is a proper interval bigraph if and only if it does not contain a bipartite claw, a bipartite net, or a bipartite tent.
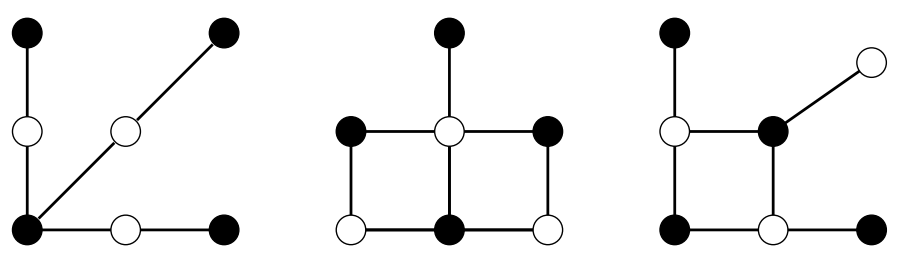

Fig. 3. The bipartite claw, net and tent

\section{The Dichotomy For Trees}

In this section, we prove an extension of Theorem 2 to graphs $H$ that contain a bipartite claw. As in the case of large cycles, we focus on the weighted version of the problem and show that it is NP-complete when the target graph $H$ contains a bipartite claw. As a corollary we will obtain our dichotomy classification for trees, Theorem 1 .

Lemma 8. Let $H$ be a fixed graph containing the bipartite claw as an induced subgraph. Then the weighted minimum constrained cost homomorphism problem to $H$ is NP-complete.

It is well known that the problem of finding a maximum independent set in a graph is NP-complete. Alekseev and Lozin citelozin proved that the problem is still NP-complete even when the input is restricted to be a 3-partite graph, cf. Gutin, Hell, Rafiey and Yeo [6].

Theorem 9. [216] The problem of finding a maximum independent set in a 3 -partite graph $G$, even given the three partite sets, in NP-complete. 
The main idea of the proof of Lemma 8 is similar to the proofs of Lemmata 4 and 6 . We show that finding an independent set of size at least $k$ in an arbitrary 3 -partite graph $G$ is equivalent to finding a homomorphism of cost at most $k^{\prime}$ in an auxiliary graph $G^{\prime}$ together with constrained costs $c$ and vertex weights $w$. To construct $G^{\prime}$, we start by adding a fixed number of placeholder vertices; vertices that, with the appropriate weights and costs, always map to the same specific vertices of the target graph $H$ in any homomorphism of $G^{\prime}$ to $H$ of minimum cost. We then use these placeholder vertices in our construction to ensure that the vertices corresponding to each part of the the input graph $G$ are only mapped to certain vertices of $H$.

Proof. The membership in NP is clear. To show that the problem is NPhard, we reduce from the problem of finding a maximum independent set in a 3-partite graph, stated in Theorem 9. Let $G$ be a a 3-partite graph in which we seek an independent set of size $k$, with parts $V_{1}, V_{2}$, and $V_{3}$, and denote by and $n$ and $m$ the number of vertices and edges in $G$, respectively. We assume that $G$ is non-empty. Without loss of generality, we can assume that $\left|V_{1}\right| \geq 1$. We construct an instance $\left(G^{\prime}, c, w, T_{G, k}\right)$ of the weighted minimum cost graph homomorphism and show that $G$ has an independent set of size $k$ if and only if there is a homomorphism of $G^{\prime}$ to $H$ with cost less than or equal to $T_{G, k}$.

We construct the bipartite graph $G^{\prime}$ as follows. Subdivide every edge $e$ in $G$ using a new vertex $d_{e}$ (which is adjacent to both ends of $e$ ). Add three vertices $b_{1}, b_{2}$ and $b_{3}$ and make each $b_{i}$ adjacent to all vertices in $V_{i}$ for $i=1,2,3$. Finally, add three more vertices $c_{0}, c_{1}$ and $c_{2}$. Make $c_{0}$ adjacent to $b_{1}, b_{2}$ and $b_{3}, c_{1}$ adjacent to $b_{1}$ and $c_{2}$ adjacent to $b_{2}$. A 3-partite graph $G$ together with its corresponding $G^{\prime}$ is depicted in Figure 4 . For future reference, we denote the set $\left\{b_{1}, b_{2}, b_{3}, c_{0}, c_{1}, c_{2}\right\}$ by $V_{4}$.

Let $H^{\prime}=(X, Y)$ be an induced subgraph of $H$ which is isomorphic to a bipartite claw with parts $X=\left\{v_{0}, v_{1}, v_{2}, v_{3}\right\}$ and $Y=\left\{u_{1}, u_{2}, u_{3}\right\}$, and edge set

$$
E^{\prime}=\left\{u_{1} v_{1}, u_{2} v_{2}, u_{3} v_{3}, u_{1} v_{0}, u_{2} v_{0}, u_{3} v_{0}\right\} .
$$

Define the homomorphism cost function $c$ as follows (see Figure 5 ).

$-c\left(v_{0}\right)=4$

$-c\left(v_{1}\right)=c\left(u_{1}\right)=1$

$-c\left(u_{2}\right)=c\left(v_{3}\right)=3$

$-c\left(v_{2}\right)=c\left(u_{3}\right)=0$

$-c(u)=160 n(m+n)$ for every other vertex $u \notin X \cup Y$

Define the vertex weights of $G^{\prime}$ as follows.

$-w\left(b_{1}\right)=w\left(c_{1}\right)=50 n(m+n)$

$-w\left(b_{3}\right)=w\left(c_{2}\right)=160 n(m+n)$

- $w\left(b_{2}\right)=w\left(c_{0}\right)=1$

- $w(u)=4(m+n)$ for every vertex $u \in V_{1}$

$-w(u)=3(m+n)$ for every vertex $u \in V_{2}$

$-w(u)=12(m+n)$ for every vertex $u \in V_{3}$ 

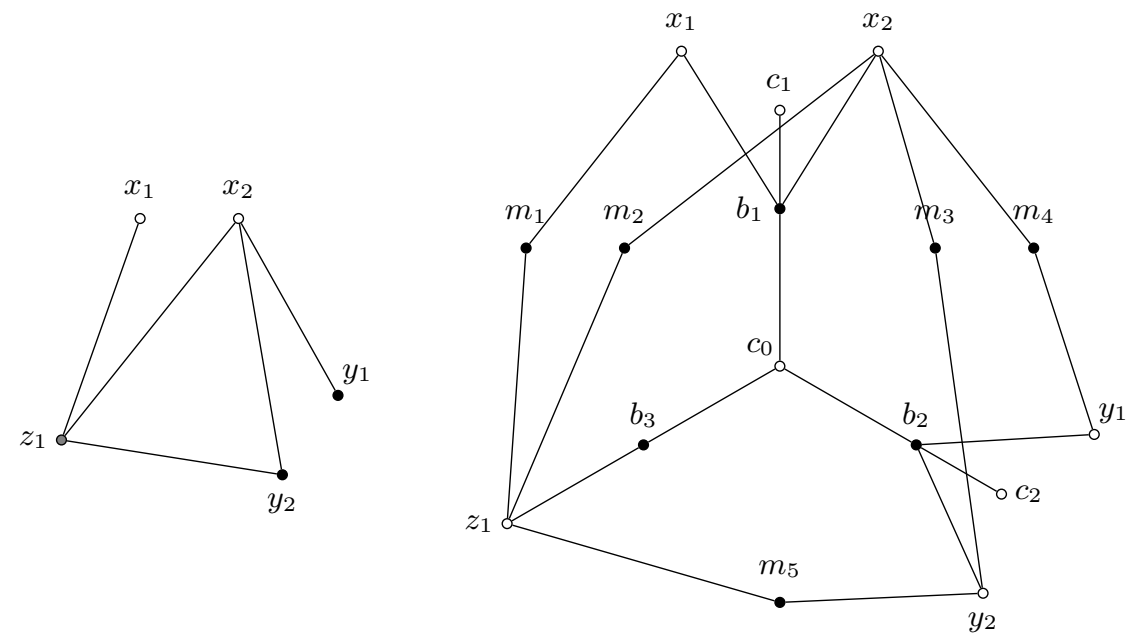

Fig. 4. A 3-partite graph $G$ with parts $V_{1}=\left\{x_{1}, x_{2}\right\}, V_{2}=\left\{y_{1}, y_{2}\right\}, V_{3}=\left\{z_{1}\right\}$ (left) and its corresponding bipartite graph $G^{\prime}$ (right)

Finally, let $T_{G, k}$ be the sum of the following values.

$-T_{G, k}^{1}=16(m+n)\left|V_{1}\right|$,

$-T_{G, k}^{2}=12(m+n)\left|V_{2}\right|$,

$-T_{G, k}^{3}=48(m+n)\left|V_{3}\right|$,

$-T_{G, k}^{4}=2 \times 50 n(m+n)+4+3$,

$-T_{G, k}^{e}=3 m$, and,

$-T_{G, k}^{I}=-12(m+n) k$.

Or equivalently:

$$
T_{G, k}=100 n(m+n)+7+3 m+\left(4\left|V_{1}\right|+36\left|V_{3}\right|\right)(m+n)+12(m+n)(n-k)
$$

We prove that $G$ has an independent set of size $k$ if and only if there is a homomorphism of $G^{\prime}$ to $H$ of cost less than or equal to $T_{G, k}$.

First, assume that $I$ is an independent set of size $k$ in $G$ with parts $I_{1} \subset V_{1}$, $I_{2} \subset V_{2}$, and $I_{3} \subset V_{3}$. Let $k_{i}$ denote $\left|I_{i}\right|(i=1,2,3)$. Define the homomorphism $f_{I}$ as follows.

- $f_{I}(u)=v_{i}$ for all vertices $u \in I_{i}(i=1,2,3)$,

- $f_{I}(u)=v_{0}$ for all vertices $u \in V(G)-I$,

- $f_{I}\left(d_{e}\right)=u_{j}$ for every edge $e$ with one end in $I_{j}(j=1,2,3)$,

- $f_{I}\left(d_{e}\right)=u_{3}$ for every edge $e$ with both ends in $V-I$,

- $f_{I}\left(b_{j}\right)=u_{j}$ for $j=1,2,3$, and finally,

- $f_{I}\left(c_{k}\right)=v_{k}$ for $k=0,1,2$. 


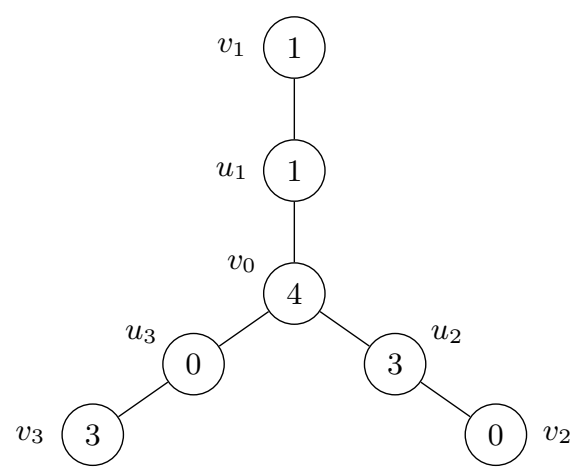

Fig. 5. A bipartite claw, with homomorphism costs

Notice that at most one end of each edge is in $I$, hence, the above assignment is indeed a function. In fact, it is easy to verify that $f_{I}$ is a homomorphism.

- edges subdivided from edges $e$ with both ends in $V-I$ map to $v_{0} u_{3}$,

- edges subdivided from edges $e$ with one end in $I_{i}$ and the other end in $V-I$ map to $u_{i} v_{i}$ and $u_{i} v_{0}(i=1,2,3)$,

- edges connecting $b_{i}$ to $V_{i}$ map to $u_{i} v_{i}(i=1,2,3)$,

- $c_{0} b_{i}$ map to $v_{0} u_{i}(i=1,2,3)$, and,

- $b_{i} c_{i}$ map to $v_{i} u_{i}(i=1,2)$.

We now compute the cost of $f_{I}$ and show that it does not exceed $T_{G, k}$.

- The vertices in $V_{1}$ contribute exactly $\left(\left|V_{1}\right|-k_{1}\right) \times 16(m+n)+k_{1} \times 4(m+n)$, or, $T_{G, k}^{1}-12 k_{1}(m+n)$,

- the vertices in $V_{2}$ contribute exactly $\left(\left|V_{2}\right|-k_{2}\right) \times 12(m+n)+k_{1} \times 0$, or, $T_{G, k}^{2}-12 k_{2}(m+n)$,

- the vertices in $V_{3}$ contribute exactly $\left(\left|V_{3}\right|-k_{3}\right) \times 48(m+n)+k_{3} \times 36(m+n)$, or, $T_{G, k}^{3}-12 k_{3}(m+n)$,

- the vertices in $V_{4}$ contribute a total of $100 n(m+n)+7=T_{G, k}^{4}$ (see Table1),

- the vertices $d_{e}$ contribute at most $3 m=T_{G, k}^{e}$.

Notice that $k=k_{1}+k_{2}+k_{3}$, hence, the cost of $f_{I}$ is at most $T_{G, k}$.

Conversely, assume that $f$ is a homomorphism of $G^{\prime}$ to $H$ which costs less than or equal to $T_{G, k}$. Note that $T_{G, k}<150 n(m+n)$. This prevents any vertex $v$ to map to a vertex $a$ when $c(v, a) \times w(v) \geq T_{G, k}$. In particular, $b_{1}$ and $c_{1}$ can only map to vertices $a$ with $c(a)<3$, i.e, $v_{1}, u_{1}, v_{2}, u_{3}$. But $b_{1}$ and $c_{1}$ are adjacent and the only edge in $H$ among these four vertices is $u_{1} v_{1}$. Similarly, $b_{3}$ and $c_{2}$ can only map to $u_{3}$ or $v_{2}$. Observe that $f\left(b_{3}\right)=v_{2}$ is not feasible, as it implies $f\left(c_{0}\right)=u_{2}$ and hence $f\left(b_{1}\right) \in\left\{v_{0}, v_{2}\right\}$. Thus, we have $f\left(b_{3}\right)=u_{3}, f\left(b_{1}\right)=u_{1}$, $f\left(c_{1}\right)=v_{1}, f\left(c_{0}\right)=v_{0}, f\left(c_{2}\right)=v_{2}$, and finally $f\left(b_{2}\right)=u_{2}$.

This restricts possible images of vertices in $V$. Specifically, all vertices in $V_{1}$ are adjacent to $b_{1}$, thus, $f$ can only map them to $v_{1}$ or $v_{0}$, the neighbourhood 


\begin{tabular}{|c|c|c|c|c|}
\hline vertex $v$ & $w(v)$ & $f_{I}(v)$ & $c\left(f_{I}(v)\right)$ & contributed cost of $v$ \\
\hline$b_{1}$ & $50 n(m+n)$ & $u_{1}$ & 1 & $50 n(m+n)$ \\
$b_{2}$ & 1 & $u_{2}$ & 3 & 3 \\
$b_{3}$ & $160 n(m+n)$ & $u_{3}$ & 0 & 0 \\
$c_{0}$ & 1 & $v_{0}$ & 4 & 4 \\
$c_{1}$ & $50 n(m+n)$ & $v_{1}$ & 1 & $50 n(m+n)$ \\
$c_{2}$ & $160 n(m+n)$ & $v_{2}$ & 0 & 0 \\
\hline
\end{tabular}

Table 1. contribution of vertices in $V_{4}$ to the cost of homomorphism $f_{I}$

of $u_{1}=f\left(b_{1}\right)$. Similarly, each vertex in $V_{2}$ will only map to $v_{2}$ or $v_{0}$, and each vertex in $V_{3}$ will only map to $v_{3}$ or $v_{0}$.

Let $I$ denote the set of vertices of $G$ that $f$ maps to $v_{1}, v_{2}$ or $v_{3}$. Notice that $I$ is an independent set in $G$. This is because any two adjacent vertices in $G$ are of distance two in $G^{\prime}$ but the shortest path between $v_{1}$ and $v_{2}$, or between $v_{2}$ and $v_{3}$, or between $v_{3}$ and $v_{1}$ in $H^{\prime}$ has length 4 .

We complete the proof by showing that $|I| \geq k$. Let $|I|=k^{\prime}$ and assume for a contradiction that $k^{\prime}<k$. Let $f_{I}$ denote the homomorphism of $G^{\prime}$ to $H$ constructed from $I$ as described in the first part of the proof with $\operatorname{cost}\left(f_{I}\right) \leq$ $T_{G, k^{\prime}}$. Observe that $f$ and $f_{I}$ are identical for every vertex $v \in V_{i}(i=1,2,3,4)$. Hence, $\left|\operatorname{cost}(f)-\operatorname{cost}\left(f_{I}\right)\right| \leq 3 m$. This implies that $\operatorname{cost}\left(f_{I}\right) \leq \operatorname{cost}(f)+3 m$. Also, note that $\operatorname{cost}\left(f_{I}\right) \geq T_{G, k^{\prime}}-3 m$, hence, we have $T_{G, k^{\prime}}-3 m \leq T_{G, k}+3 m$, or equivalently, $T_{G, k^{\prime}}-T_{G, k} \leq 6 \mathrm{~m}$. But this is a contradiction because:

$$
T_{G, k^{\prime}}-T_{G, k}=T_{G, k^{\prime}}^{I}-T_{G, k}^{I}=12(m+n)\left(k-k^{\prime}\right) \geq 12(m+n) .
$$

We can now apply Theorem 3 and derive the same conclusion for the problem without vertex weights.

Theorem 10. Let $H$ be a fixed graph containing the bipartite claw as an induced subgraph. Then the minimum constrained cost homomorphism problem to $H$ is NP-complete.

Note that Lemma 7 implies that for trees, a chordal bipartite $H$ is a proper interval bigraph if and only if it does not contain an induced bipartite claw. Thus we obtain Theorem 1 as a corollary.

\section{Conclusion}

We left open the complexity of the minimum constrained cost graph homomorphism problems in general. In particular, it remains to check whether the problem is NP-complete also for graphs $H$ that contain a bipartite net or a bipartite tent. 


\section{References}

1. P. Hell and J. Nešetřil, On the complexity of $H$-coloring, J. Comb. Theory, Ser. B, 48 (1990) $92-110$.

2. T. Feder, P. Hell, and J. Huang, List homomorphisms and circular arc graphs, Combinatorica 19 (1999) $487-505$.

3. G. Gutin, A. Rafiey, A. Yeo, M. Tso Level of repair analysis and minimum cost homomorphisms of graphs, Discrete Appl. Math. 154 (2006) 881 - 889.

4. Amotz Bar-Noy, Guy Kortsarz, Minimum Color Sum of Bipartite Graphs, J. Algorithms, 28 (1998) $339-365$.

5. K. Supowit, Finding a maximum planar subset of a set of nets in a channel, IEEE Trans. Computer-Aided Design 6 (1987) 93 - 94.

6. G. Gutin, P. Hell, A. Rafiey and A. Yeo, A dichotomy for minimum cost homomorphisms, European J. Comb. 29 (2008) 900 - 911.

7. Tomás Feder and Moshe Y. Vardi, The Computational Structure of Monotone Monadic SNP and Constraint Satisfaction: A Study through Datalog and Group Theory, SIAM J. Comp. 28 (1998) $57-104$.

8. A. Bulatov, P. Jeavons and A. Krokhin, Classifying the Complexity of Constraints Using Finite Algebras, SIAM J. Comp., 34(3), 720 - 742.

9. T. Feder, P. Hell, and J. Huang, Bi-arc graphs and the complexity of list homomorphisms, J. Graph Th. 42 (2003) $61-80$.

10. Bulatov, A., Complexity of Conservative Constraint Satisfaction Problems, ACM Trans. Comput. Logic 12 (2011) 24:1 - 24:66.

11. P. Hell and A. Rafiey, The Dichotomy of Minimum Cost Homomorphism Problems for Digraphs, SIAM J. Disc. Math. 26(4) 1597 - 1608.

12. P. Hell and A. Rafiey, The dichotomy of list homomorphisms for digraphs, in Proceedings of the Symposium on Discrete Algorithms SODA 2011, pp. 1703 1713.

13. P. Hell and A. Rafiey, Duality for Min-Max Orderings and Dichotomy for Min Cost Homomorphisms, arXiv preprint (2009) arXiv:0907.3016

14. P. Hell and A. Rafiey, Minimum Cost Homomorphism Problems to Smooth and Balanced Digraphs, manuscript, 2007.

15. Rustem Takhanov, A Dichotomy Theorem for the General Minimum Cost Homomorphism Problem, 27th International Symposium on Theoretical Aspects of Computer Science 5 (2010) 657 - 668.

16. Kolmogorov, V. and Živný, S., The Complexity of Conservative Valued CSPs, J. ACM 60 (2013) 10:1 - 10:38.

17. H. Müller, Recognizing interval digraphs and interval bigraphs in polynomial, Discrete Applied Mathematics 78 (1997) 189 - 205.

18. J. Spinrad, A. Brandstädt, and L. Stewart, Bipartite permutation graphs, Discrete Applied Mathematics 18 (1987) 279 - 292.

19. T. Feder, P. Hell List Homomorphism to reflexive graphs, J. Combin. Theory B 72 (1998) $236-250$.

20. P. Hell and J. Huang, Interval bigraphs and circular arc graphs, J. Graph Theory 46 (2004) $313-327$.

21. V.E. Alekseev and V.V. Lozin, Independent sets of maximum weight in (p,q)colorable graphs, Discrete Mathematics 265 (2003) $351-356$. 